\title{
Least Significant Bit Image Steganography using Particle Swarm Optimization and Optical Pixel Adjustment
}

\author{
Parisa Gerami \\ Faculty of Computer Science \& \\ Information System \\ Universiti Teknologi Malaysia \\ 81310 UTM Skudai \\ Johore, MALAYSIA
}

\author{
Subariah Ibrahim \\ Faculty of Computer Science \& \\ Information System \\ Universiti Teknologi Malaysia \\ 81310 UTM Skudai \\ Johore, MALAYSIA
}

\author{
Morteza Bashardoost \\ Faculty of Computer Science \& \\ Information System \\ Universiti Teknologi Malaysia \\ 81310 UTM Skudai \\ Johore, MALAYSIA
}

\begin{abstract}
Data hiding is a method of concealing secret data into a cover media and preventing a spectator from being aware of the existence of the hidden message. According to the problems of steganography, the main effort is to provide a better imperceptibility of stego-image that can be done by decreasing distortion of image. One of the popular techniques in data hiding is steganography in which the simple method for image hiding is the Least Significant Bit (LSB) substitution method. Wu et al. proposed two image hiding techniques to improve the quality of the stego-image [1]. The first one is to find the best block matching matrix and the other one is to find the optimal substitution matrices. The proposed method utilizes particle Swarm optimization (PSO) for finding the best pixel locations, and then the secret image is transformed to a new secret image. Optimal Pixel Adjustment (OPA) method is applied to further increase the quality of image. Results are then compared with those obtained by Simple LSB, Wang's et al. method, Wu's et al. method. The experimental results confirmed that PSNR of the proposed method is higher than mentioned methods which mean the imperceptibility of the image improves. Also the results illustrate that the proposed approach is robust against chi-square attack.
\end{abstract}

\section{Keywords}

Data hiding, image steganography, Particle Swarm optimization.

\section{INTRODUCTION}

Nowadays people tend to exchange large amounts of data through internet. However, transferring data in network will increase the risk of unlawful and unauthorized access. Data hiding is a key to safe communication. It is a way of concealing secret data into a cover media and preventing any user from being aware of the existence of the hidden message. Steganography is one of the popular techniques in data hiding, it is the art of hiding secret data in media files such as audio and different types of image formats, so it does not attract any observer attention and look like a simple medium[2].

In image hiding, the secret data is concealed in cover image and the result is called a stego-image while its quality should be acceptable so as not to observe the attraction of other people. A number of steganography approaches exist for embedding data in an image which can be classified as transform domain technique and special domain technique[3].
The simplest and most popular technique in special domain is least significant bit which embeds data in the LSB position of bit pixel of a cover image. Researchers proposed many image hiding techniques based on LSB. For example a new method of LSB uses Genetic Algorithm (GA) proposed by Wang et $a l$. in which the purpose of using GA is to find an optimal replacement of LSB in order to increase the imperceptibility[4].

$\mathrm{Wu}$ et al. used two approaches to enhance the quality of stego-image and also to increase the hiding capacity of stegoimage[1]. These approaches are called global method and local method. In Wu's et al. approach, GA was used for finding the desired matrices for transferring the blocks and value pixel of secret image to a new secret image based on comparing cover image and secret image. Another method is optimal pixel adjustment (OPA) that modifies simple LSB, the aim of which is to have a stego-image with a better quality[5].

According to the problems of steganography, the main effort is to provide a better imperceptibility of stego-image that can be done by decreasing image distortion. The proposed algorithm is an improvement of Wu et al. global and local substitution strategies that utilize PSO rather than GA. The quality of stego-image is improved by combining proposed method with OPA. By using the proposed method, better image quality will be obtained and image distortion will be decrease. Furthermore, the hiding capacity in this study depends on the secret image size and also the size of the cover image that will be embedded.

\section{RELATED WORK}

\subsection{Simple LSB Substitution}

The most common and easiest way of concealing secret data is LSB substitution method. The primary concept of LSB is to conceal the secret in the least significant bit of the image[6]. For example, for hiding image $\mathrm{E}$ into image $\mathrm{H}$ which we assume both of them to be n-bit grayscale images, a suitable technique is to conceal $\mathrm{E}$ into the position of the least significant bit of $\mathrm{H}$. At first, the right most $\mathrm{k}$ LSBs from each pixel of $\mathrm{H}$ are extracted to create a k-bit gray-scale image, $\mathrm{R}$, called the residual image which equals $\mathrm{E}$ in size. Next, $\mathrm{E}$ is changed to a k-bit image by disintegrating each pixel of $\mathrm{E}$ into various small $\mathrm{k}$-bit units and processing each unit as a single pixel. The result of this decomposition is E'. Eventually, $\mathrm{R}$ is replaced by E'. The embedding result is $\mathrm{Z}$ which is illustrated in Figure 1. 


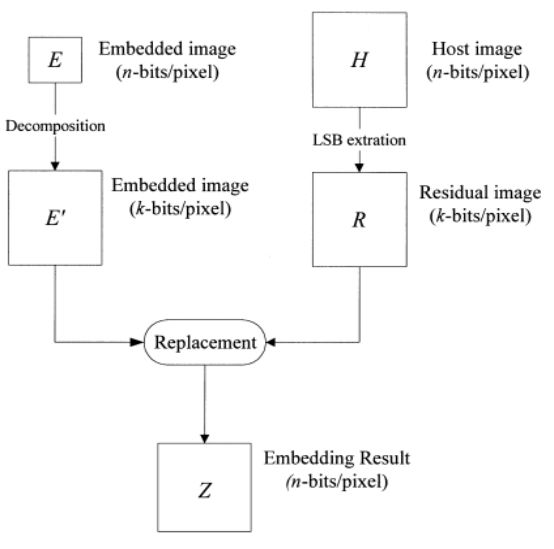

Fig.1.Simple LSB substitution

\subsection{Wu's et al. Method}

In Wu's et al. methods, the transformation matrices are computed according to the characteristic of blocks of cover image and the result of this transformation is shown that using the optimal substitution matrix for each block will increase peak signal to noise ratio (PSNR) value.

In the method pursued by Wu et al. two various approaches have been suggested. The first method is the global optimal substitution and the second one is local optimal substitution. Global method needs the same substitution matrix for whole blocks, so it records fewer amounts of data. Local method uses various substitution matrices for every block; therefore, it demands more matrices. Since the local method requires more matrices, in this approach the stego-image in this approach will provide a superior image quality than the global one. Figure 2 illustrates the block diagram of the proposed method in more detail.

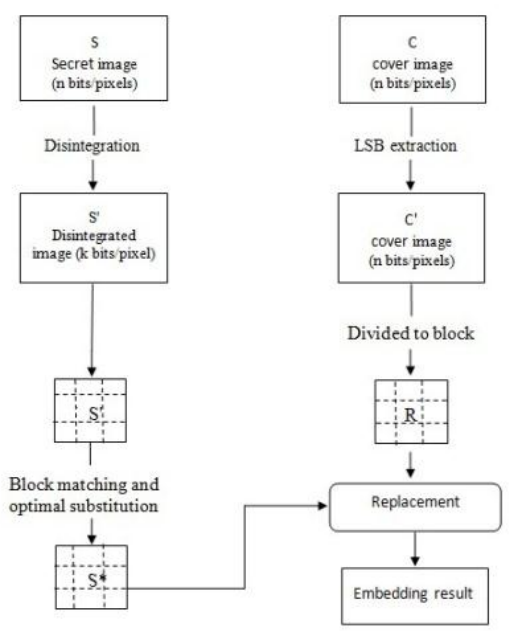

Fig.2. Block diagram of Wu's et al. global

\subsection{Optimal Pixel Adjustment}

Pixel adjustment process (OPAP) has been suggested to increase the quality of the stego-image which is acquired by the LSB substitution technique[7]. The main idea of applying OPAP is to minimize the errors of the cover image and the embedded image. In this method, the values of pixels are modified after concealing secret image into cover image in order to enhance the quality of the stego-image without disturbing the data hidden. The hiding procedures are shown in the following steps. At first, the least significant bits of the cover image are embedded by the secret image. Then those bits which did not changed in the embedding process like the most significant bits will be modified by this method if it provides less error. Here $n$ is the $n$ Least Significant Bits that are substituted in each pixel and $d$ is the value of the pixel after the substitution, so $\mathrm{d} 1$ is the value of last $\mathrm{n}$ bits of the pixel and $d 2$ is the value of $\mathrm{n}$ bits hidden in that pixel. In the end, $\mathrm{d}$ is converted to binary and written back to the pixel. Let $C_{\mathrm{i}}, S_{i}, P_{i}$ be the pixel values of the $i$ 'th pixel of cover image, the stego-image which is achieved by simple LSB substitution method and the output which is the stego-image after using OPA algorithm, respectively. Let $\delta=S_{i}-C_{i}$ be the embedding error between the cover image and stegoimage. Based on the value of $\delta, P_{i}$ will change according to three conditions. These three conditions are illustrated as follows:

Case $1,(2 k-1<\delta<2 k)$ :

if $S \geq 2 k$, then $P_{i}=S_{i}-2 k$ otherwise $P_{i}=S$;

Case $2,(-2 k-1<\delta<2 k-1): P_{i}=S$;

Case $3(-2 k-1<\delta<-2 k-1)$ :

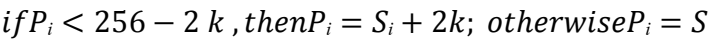

For reducing the error between cover image and stego-image and obtaining better image quality, OPA method is used after embedding with simple LSB substitution according to $\mathrm{Wu}$ 's et al. method.

The four fields that comprise the area of soft computing are fuzzy computing, evolutionary computing, artificial neural networks, and probabilistic computing. Genetic Algorithm and Particle swarm optimization are subcategories of evolutionary computing which have been used in steganography problems. PSO has a lot of resemblance and many differences with GA. The system in PSO begins with a population of haphazard locations and it will look for an optimum via updating generations and it does not have evolution operators such as crossover and mutation. The particles fly through the problem area by following the current optimum particle.GA is considered as a randomized search procedure that is used to solve the optimization problems. Wang et al.[4] used GA for finding the optimal substitution matrices for transforming the secret image to a new secret image which produces a stego-image with high peak signalto-noise ratio (PSNR) and also diminish the search time. Wu et al. also used GA for the same purpose of Wang's et al. method to increase the quality of the embedded image. PSO has been used by Bedi et al. [8]for discovering the optimal pixel position in the cover image for hiding the secret image. In other words they used PSO to find the similarity between the cover image and the secret image in order to construct less distortion. A lot of optimization problems have been solved by PSO and the results illustrate that PSO is effective and quick. It was successfully applied in many research and application areas. In this method PSO is used for detecting the optimal substitution matrices. 


\section{THE PROPOSED METHOD}

\subsection{Process of the Method}

Great numbers of techniques were proposed by researchers to increase the quality of stego-image such as simple LSB substitution and Wu's et al method. The suggested approach will provides a stego-image with better quality which is unnoticeable by intruders. The process of performing the proposed method is explained in four steps. These four steps are as follows:

i. Transforming secret image and cover image

ii. Reshuffle secret image according to Wu's et al. method

iii. Embedding secret image into 4 bit LSB of cover image

iv. Applying OPA on stego-image

For better understanding of the proposed method, assume the cover image $\mathrm{H}$ and secret image $\mathrm{S}$ are 8 -bit grayscale images. The size of cover image is $512 \times 512$ and the size of secret image is $512 \times 256$. $\mathrm{K}$ is the number of embedding bits which is 4 in this example. Cover image is partitioned into two parts, the rightmost $\mathrm{k}=4$ bits of each pixel of the cover image is retrieved to constitute the Residual image and the (n-k) leftmost bits of each pixel is also retrieved to form the MSB. Secret image $S$ is decomposed to a new secret image $S^{\prime}$ with size $512 \times 512$. Each pixel of $S^{\prime}$ is 4 bit value of each pixel of $\mathrm{S}$. Figure 3 shows the process of decomposing secret image to $\mathrm{S}^{\prime}$ and dividing cover image to $\mathrm{R}$ and $\mathrm{L}$.

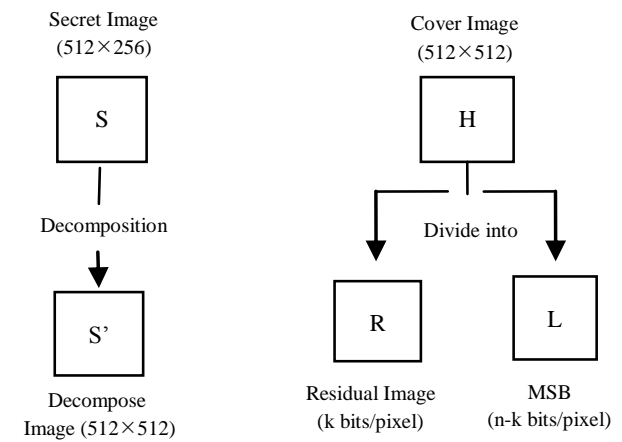

Fig. 3: Transferring of secret image and cover image

In order to increase the imperceptibility of stego-image, $\mathrm{Wu}$ et al. proposed a method which finds the best transformation matrices for substituting blocks or pixel values of secret image by a new secret image by comparing the cover image with secret image. By this method, the secret image is changed according to cover image, hence the data is embedded in a better place and the quality of image is increased. This step includes two methods for finding the matrices that is explained in the following and is implemented respectively. The first method is to search for the best block matching matrix and the second one is to search for different optimal substitution pairs.

\subsection{First: Best Block Matching Matrix}

For finding block matching matrix, $\mathrm{S}^{\prime}$ and $\mathrm{R}$ are partitioned into X blocks $\left\{S_{1}^{\prime}, S_{2}^{\prime}, S_{3}^{\prime}, \ldots S_{x}^{\prime}\right\},\left\{R_{1}, R_{2}, R_{3}, \ldots R_{\chi}\right\}$. PSO is used to search for this matrix. It will find the most similar block between $\mathrm{S}^{\prime}$ and $\mathrm{R}$. These can be divided to 4, 8, 16 block. Figure 4 will show the flowchart of comparing $S^{\prime}$ and R.

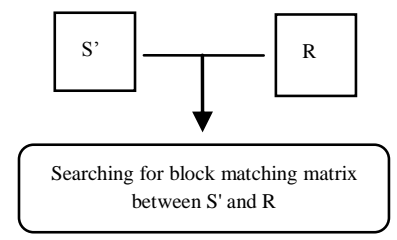

Figure.4: Flowchart of comparing $S^{\prime}$ and $R$

\subsection{Second: Optimal Substitution Matrix}

After finding the block matching matrix, two other strategies have been proposed for finding optimal substitution. The first one is called global optimal substitution method and the second one is called local optimal substitution method. PSO is used for finding the optimal matrices, the secret image blocks will transform to new location base on the block matching matrix and then according to the global optimal substitution or local optimal substitution. The pixel value of secret image is transformed to new pixel value. After applying theses matrices to secret image $S^{\prime}$ the $S^{\prime \prime}$ is obtained which will replaces by $R$. $T$ is the least significant bit of cover image that are replaced by secret image and $\mathrm{Z}$ is the result of merging $\mathrm{T}$ and $\mathrm{L}$ to gain the embedded image. It means that the secret image is embedding in LSBs of cover image. The name of this method is simple LSB substitution as explained in the previous section. The flowchart in Figure 5 shows the flow of the proposed method. By LSB the secret is embedded in the least significant bit and the number of embedding determine the payload size which is 4 bits in this method. By embedding 4 bits of secret image to cover image, the quality of cover image is reduced and provide a higher distortion. Optimal pixel adjustment (OPA) has been proposed by Chan et al. This method increases the imperceptibility of cover image without changing the least significant bit of cover image.

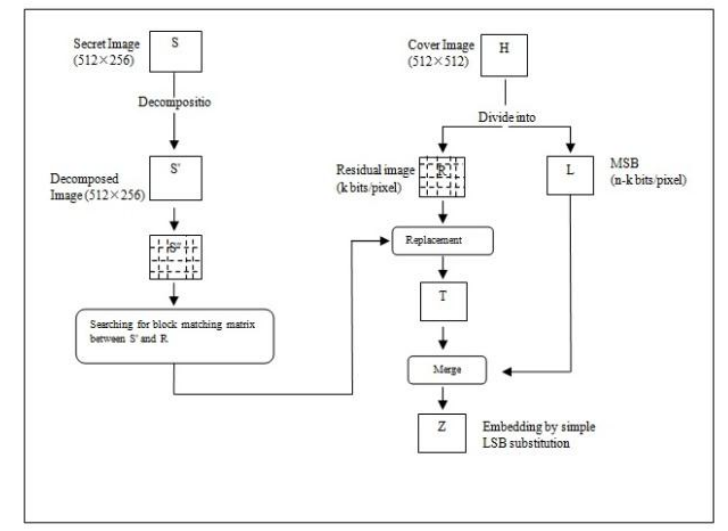

Fig.5: Proposed method flowchart

The main idea of OPA is to reduce the error between cover image and stego-image. In figure 6 the flow chart of proposed method with OPA technique is shown. 


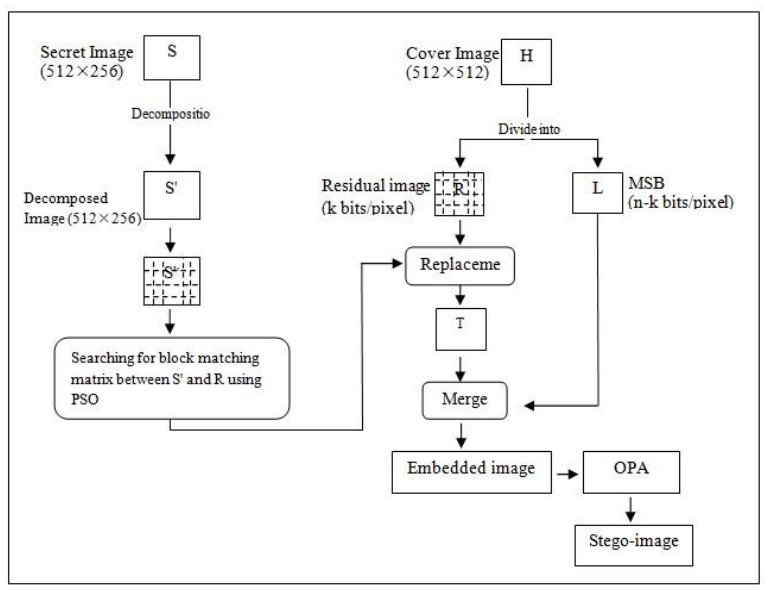

Figure.6: Proposed method flowchart with OPA

\section{EXPERIMENTAL RESULTS}

\subsection{Result of PSNR}

In this project, analysis on the proposed algorithm was conducted on cover images Lena, Baboon and secret images was Peppers, Barbara and airplane. The secret image embedded in the rightmost 4 bits of each pixel in the cover image. These images are used because $\mathrm{Wu}$ et al and Wang et $a l$ also used the same cover images and secret images and the result of the proposed algorithm were compared with these previous works. Four bits of least significant bit of cover image are utilized for embedding secret image. The result of our proposed method is compared with the existing method. The value of PSNR also used as a metric for comparing the proposed method which is the same as the metric of evaluation in the mentioned method.

In order to evaluate the proposed algorithm with Simple LSB, Wang et al.'s method and Wu et al.'s method, PSNR value of stego-image of proposed method are measured and listed in different tables for each cover image. In this project, cover image were $512 \times 512,8$ bit image with 256 grayscale and two different cover images Lena and Baboon were used which are shown in Figure 7 . Three secret images with size $512 \times 256$ were used which are Airplane-F16, pepper and Barbara that are shown in Figure 8.

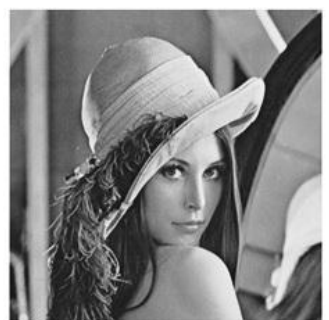

(a) Lena

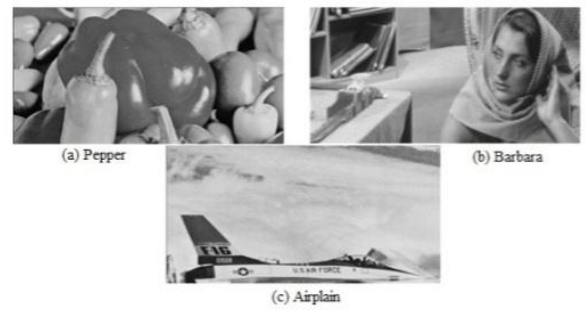

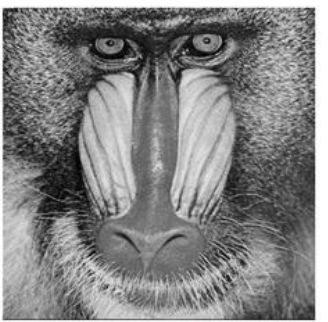

(b) Baboon
PSNR formula is used to measure the quality of image of this study to compare with other works. It is the most popular measurements that are used in all steganography works. Because many signals have a very wide dynamic range, PSNR is usually expressed in terms of a logarithmic decibel scale. The formula is as follows:

$$
\begin{aligned}
& P S N R=10 \log _{10} \frac{255^{2}}{M S E} d B \\
& M S E=\left(\frac{1}{M * N}\right) \sum_{x=0}^{M-1} \sum_{y=0}^{N-1}\left(P(x, y)-P^{\prime}(x, y)\right)^{2}
\end{aligned}
$$

If the PSNR value of stego-image is more than $36 \mathrm{~dB}$, it shows that it is extremely difficult to distinguish the differences between the original image and embedded image by an ordinary person.

The embedding result of global optimal substitution with 4 blocks in secret images are shown Table 1 The result of this table shows that value of PSNR of the proposed method in global substitution matrix by applying OPA is higher than Simple LSB. Higher value of PSNR represents better quality of stego-image; therefore the imperceptibility of stego-image by proposed method is better than Simple LSB. Comparing PSNR value of Wang et al. 's method with proposed method, illustrate that value of PSNR is improved. This method provides better imperceptibility in comparison with simple LSB and Wang et al.'s method.

Table 1. Comparison of proposed method with Simple LSB and Wang et al. in Lena

\begin{tabular}{|c|c|c|c|}
\hline $\begin{array}{c}\text { Secret } \\
\text { image }\end{array}$ & $\begin{array}{c}\text { Simple } \\
\text { LSB }\end{array}$ & $\begin{array}{c}\text { Wang's } \text { et } \\
\text { al. } \text { method }\end{array}$ & $\begin{array}{c}\text { Proposed Global } \\
\text { method }\end{array}$ \\
\hline Airplane & 31.9406 & 33.0296 & 34.8562 \\
\hline Pepper & 32.3928 & 32.5453 & 34.8266 \\
\hline Barbara & 32.3117 & 32.8824 & 34.8619 \\
\hline
\end{tabular}

The embedding result of global optimal substitution and local optimal substitution with 4 blocks in secret images are shown Table 2.From the results listed in Table 2, we can understand that our proposed method in global substitution matrix gives better PSNR. Obtained value of PSNR from this method in global method is noticeably greater than Wu's et al. approach in global method. But the value which is gained from the proposed local method has not changed much and is not noticeable. But in general the PSNR has increased in our method; therefore, it provides better quality of stego-image. It is hard to understand that a secret image embedded in this image and prevent unauthorized person to be aware of the secret data.

Table2. Comparison of proposed method with $\mathrm{Wu}$ et al. in Lena

\begin{tabular}{|c|c|c|c|c|}
\hline $\begin{array}{c}\text { Secret } \\
\text { image }\end{array}$ & $\begin{array}{c}\text { Wu } \text { et al. } \\
\text { global }\end{array}$ & $\begin{array}{c}\text { Wu } \text { et al. } \\
\text { local }\end{array}$ & $\begin{array}{c}\text { Proposed } \\
\text { global }\end{array}$ & $\begin{array}{c}\text { Proposed } \\
\text { local }\end{array}$ \\
\hline Airplane & 33.1669 & 34.5349 & 34.8562 & 34.8527 \\
\hline Pepper & 32.6748 & 34.3667 & 34.8266 & 34.9002 \\
\hline Barbara & 32.9873 & 34.3948 & 34.8619 & 34.8232 \\
\hline
\end{tabular}

Fig .7: The cover images and the secret images 
The embedding result of global optimal substitution with 16 blocks in secret images is shown in Table 3. The results of implanting secret images in cover image Baboon is shown in this table. It is quite evident from the PSNR value that our method ends in better value than Simple LSB and the method used by Wang et al. PSO finds the best place for embedding secret image in cover image by considering minimum MSEs of secret image and cover image.

Table 3. Comparison of proposed method with Simple LSB and Wang et al. in Baboon

\begin{tabular}{|c|c|c|c|}
\hline $\begin{array}{c}\text { Secret } \\
\text { image }\end{array}$ & $\begin{array}{c}\text { Simple } \\
\text { LSB }\end{array}$ & $\begin{array}{c}\text { Wang's } \text { et } \\
\text { al. } \text { method }\end{array}$ & $\begin{array}{c}\text { Proposed Global } \\
\text { method }\end{array}$ \\
\hline Airplane & 32.0185 & 32.8717 & 34.4772 \\
\hline Pepper & 32.4772 & 32.8512 & 34.8506 \\
\hline Barbara & 32.3864 & 35.7586 & 34.8471 \\
\hline
\end{tabular}

The results in Table 4 are provided to show the comparison between Wu's et al. method and our proposed approach with 16 blocking. By comparing the value of PSNR in global method, we can understand that the PSNR increased in the all three secret images. But the PSNR value for local method depicts that PSNR has not changed much.

Table 4. Comparison of proposed method with Wu et al. in Baboon

\begin{tabular}{|l|c|c|c|c|}
\hline \multicolumn{1}{|c|}{$\begin{array}{c}\text { Secret } \\
\text { image }\end{array}$} & $\begin{array}{c}\text { Wu } \text { et al. } \\
\text { global }\end{array}$ & $\begin{array}{c}\text { Wu } \text { et al. } \\
\text { local }\end{array}$ & $\begin{array}{c}\text { Proposed } \\
\text { global }\end{array}$ & $\begin{array}{c}\text { Proposed } \\
\text { local }\end{array}$ \\
\hline Airplane & 33.0109 & 34.2259 & 34.8506 & 34.8450 \\
\hline Pepper & 32.9987 & 34.1418 & 34.8461 & 34.8584 \\
\hline Barbara & 32.0010 & 34.2859 & 34.8471 & 34.8396 \\
\hline
\end{tabular}

\subsection{Security of Proposed Method}

Chi square attack is very simple and has been used to prove the security of proposed method. The goal of this attack is to show the probability of distribution of zeros and ones in stego-image and find the statistical evidence that is left by the embedding process[9]. The result of probability will be 50 percent that means half of the numbers of bits are zero or one when the bits generates randomly in secret message. Pair of value (POV) is one of the results of an embedding algorithm which means the values of the pixel were embedded into one another. POV is a detection algorithm which is designed to perform chi-square attack on image and the output is the probability of embedding[10].

Assume $\mathrm{X}^{128}$ and $\mathrm{Y}^{128}$ represent two vectors such that $\mathrm{x}_{\mathrm{i}}=$ frequency $(2 \mathrm{i})$ and $\mathrm{y}_{\mathrm{i}}=$ frequency $(2 \mathrm{i}), 0 \leq \mathrm{i} \leq 127$. Then the gray scale values are counted by the algorithm in image and increase the corresponding entry in $\mathrm{X}$ or $\mathrm{Y}$. Expected frequency of gray values of $2 \mathrm{i}$ and $2 \mathrm{i}+1$ are existent as:

$$
\mathrm{z}_{\mathrm{i}}=\frac{\mathrm{x}_{\mathrm{i}}+\mathrm{y}_{\mathrm{i}}}{2}
$$

Presume that there are $\mathrm{n}$ categories. There are 128 categories $(256 / 2)$ when the image is 8-bit gray scale image. The chi- square statistic is given as $x_{n-1}^{2}$ with $n-1$ degrees of freedom which are calculated as:

$$
X_{n-1}^{2}=\sum_{j=0}^{127} \frac{\left(x_{j}-z_{j}\right)}{z_{j}}
$$

Where $\mathrm{Z}_{\mathrm{j}}$ is calculated as:

$$
\mathrm{z}_{\mathrm{j}}=\frac{\mathrm{x}_{\mathrm{j}}+\mathrm{y}_{\mathrm{j}}}{2}
$$

For a stego-image $X_{n-1}^{2}$ is relatively small because $x_{j}$ should be near $z_{j}$ and for a non stego image $X_{n-1}^{2}$ is relatively large because $x_{j}$ should be far fromz $z_{j}$. The last step for chi-square is to calculating $\mathrm{p}$ which is the probability of embedding by merging the density function with $\mathrm{X}_{\mathrm{n}-1}^{2}$ as it upper limit:

$$
\mathrm{p}=1-\frac{1}{2^{\frac{n-1}{2}} \Gamma\left(\frac{\mathrm{n}-1}{2}\right)} \int_{0}^{\mathrm{x}_{\mathrm{n}-1}^{2}} \mathrm{e}^{-\frac{\mathrm{u}}{2}} \mathrm{u}^{\frac{\mathrm{n}-1}{2}-1} \mathrm{du}
$$

If $\mathrm{x}_{\mathrm{j}}=\mathrm{z}_{\mathrm{j}}$ for all $\mathrm{j}$ in chi-square statistic, the probability of embedding is the probability of $X_{n-1}^{2}$.

The result of this attack is stronger and reliable when the embedding is done and more bit embedded in stego-image, for example the result of probability of embedding is one for the whole stego-image when 100 percentages of LSBs are embedded by random bits and it shows the best. Figure 8 and 9 shows the result of chi-square attack in cover image Lena and Baboon by Simple LSB substitution. The charts illustrate the existence of the message can be easily detected.

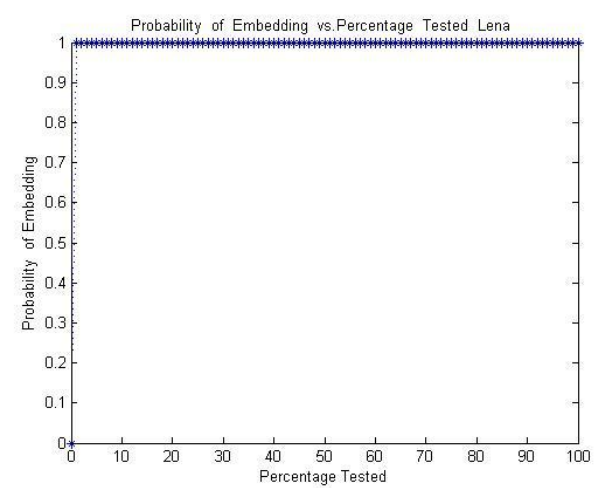

Figure.8.Result of chi-square attack on stego-image Lena embedded by simple LSB

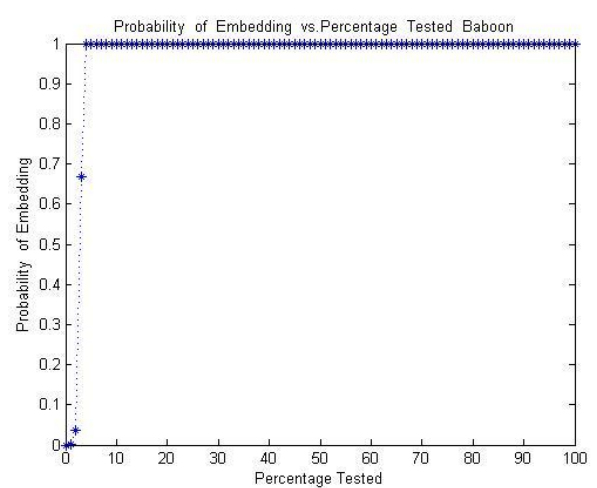

Figure.9.Result of chi-square attack on stego-image Baboon embedded by simple LSB 
The results are noticeably different when the proposed method used for embedding data into the same cover image. Figure 10 and 11 shows that the chi-square attack almost did not detect any embedded data in the stego-images. The payload capacity for this image is 4 bit in the least significant bit of cover image.

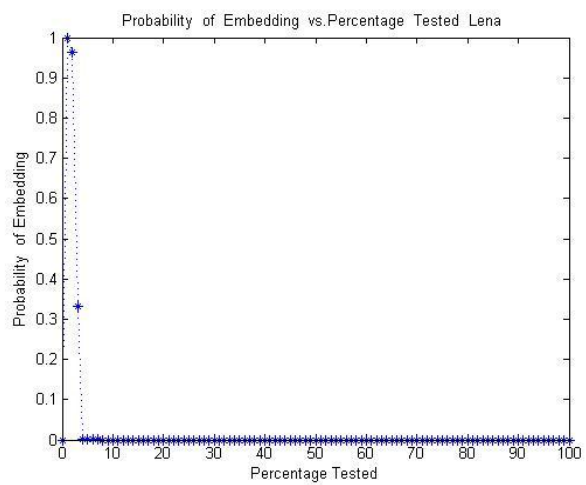

Figure.10.Result of chi-square attack on stego-image Lena embedded by proposed method

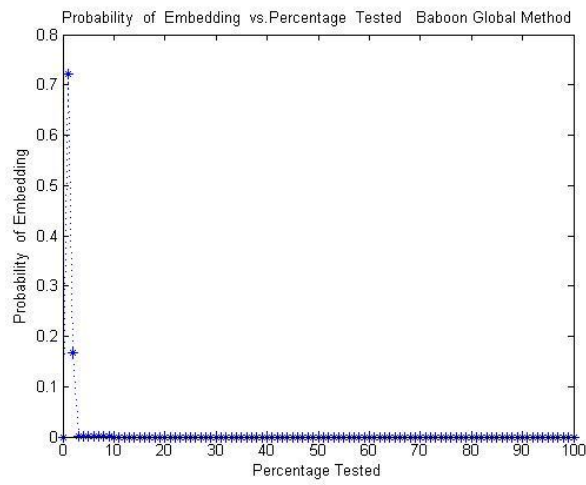

Figure.11.Result of chi-square attack on stego-image Baboon embedded by proposed method

This attack was applied in the two stego-images which the cover image was Lena and Baboon in the Local and Global proposed method. The diagrams of stego-images show that the proposed approach is strongly robust against chi-square attack.

\section{CONCLUSION}

A new approach is presented by using Wu's et al method and PSO. Also OPA technique has been used in order to decrees the distortion of the image. A comparison was undertaken between the proposed method and other previous methods and the results show that our method provides better PSNR. The quality of image did not change very much and achieved better imperceptibility with the same payload capacity. Because of the better quality of stego-image, it makes it hard for unauthorized person to recognize that data has been embedded in the image. In this approach the secret image divided into 4 and 16 blocks and compared to cover image for finding the best block matching matrices that provided higher PSNR. In the future work the secret image can partition into $64,256,1024$ blocks. If the number of blocks increases, each block of secret image will have a lot of choices for replacement according to cover image. Therefore the possibility of finding a block of cover image which is more resembled to secret image will increase. As a result the secret image will transform to a new place which obtain better
PSNR after embedding. Also finding a new method for embedding the 4 bit of secret image in the cover image with same size is another future approach in this technique. By increasing the payload capacity, large quantity of secret bits can embed in cover image.

\section{AKNOWLEDGMENTS}

This work was supported by Universiti Teknologi Malaysia (UTM), Johor, Malaysia under the VOT: Q.J13000.7128.00J29

\section{REFERENCES}

[1] Wu, M.-N., M.-H. Lin, and C.-C. Chang, A LSB Substitution Oriented Image Hiding Strategy Using Genetic Algorithms Content Computing, 2004, Springer Berlin / Heidelberg. p. 219-229.

[2] Mathkour, H., B. Al-Sadoon, and A. Touir A New Image Steganography Technique, in IEEE. 2008. p. 1-4.

[3] Chandramouli, R., M. Kharrazi, and N. Memon, Image Steganography and Steganalysis: Concepts and Practice

[4] Digital Watermarking, T. Kalker, I. Cox, and Y. Ro, Editors. 2004, Springer Berlin / Heidelberg. p. 204-211.

[5] Wang, R.-Z., C.-F. Lin, and J.-C. Lin, Image hiding by optimalLSBsubstitution and genetic algorithm. Pattern Recognition, 2001: p. 671-683.

[6] Zanganeh, O. and S. Ibrahim, Adaptive Image Steganography Based on Optimal Embedding and Robust Against Chi-square Attack. Information Technology Journal, 2011. 10: p. 1285-1294.

[7] Walia, E., P. Jain, and N. Navdeep, An Analysis of LSB \& DCT based Steganography. Global Journal of Computer Science and Technology, 2010. 10: p. 4-8.

[8] Chan, Y.-K., Y.-A. Ho, and Y.-P. Chu, Image Hiding with an Improved Genetic Algorithm and an Optimal Pixel Adjustment Process, in IEEE. 2008. p. 320-325.

[9] Bajaj, R., P. Bedi, and S. Pal, Best Hiding Capacity Scheme for Variable Length Messages Using Particle Swarm Optimization Swarm, Evolutionary, and Memetic Computing,. 2010, Springer Berlin / Heidelberg. p. 230-237.

[10] Westfeld , A. and A. Pfitzmann, Attacks on Steganographic Systems - Breaking the Steganographic Utilities EzStego in Citeseer. 2000. p. 1-4.

[11] Stanley, C.A., Pairs of Values and the Chi-squared Attack, in CiteSteer. 2005. p. 1-45. 